\title{
Estimation de la contamination radioactive individuelle par interprétation sélective des résultats de la surveillance radiotoxicologique
}

\author{
J. PIECHOWSKI*, B. MENOUX**
}

(Manuscrit reçu le 11 octobre 1994,
révisé le 11 janvier 1995, accepté le 19 janvier 1995)

RÉSUMÉ Les méthodes d'interprétation dont on dispose pour évaluer la dose individuelle à partir des résultats de mesure présentent des difficultés liées à l'adaptation des modèles métaboliques aux situations rencontrées en pratique. Ces difficultés sont essentiellement en rapport avec la définition de paramètres propres aux cas rencontrés et, bien souvent - hors incident caractérisé - à la méconnaissance de la chronologie des épisodes et des voies de contamination. Cette constatation conduit à envisager une simplification de l'interprétation des données de la surveillance, en considérant d'une part les résultats propres aux portes d'entrée et d'autre part ceux relatifs à la contamination systémique, c'est-à-dire après transfert des radionucléides vers le sang. Dans un premier temps, l'étude propose une approche pour l'interprétation des résultats de mesure relatifs à la contamination systémique. La méthode consiste à calculer, à partir de ceux-ci, les valeurs de l'activité absorbée quotidiennement depuis les portes d'entrée vers le sang, en utilisant les fonctions de rétention et d'excrétion appropriées. Un suivi de l'activité absorbée, au jour le jour, devient possible, autorisant ainsi un bilan en temps réel, facile à consulter. Quelques applications de la méthode sont proposées sur des cas de contamination aiguë au tritium et à l'uranium, et sur des contaminations chroniques au tritium, à l'uranium et à l'iode. Les conditions et contraintes pour la validité de l'approche proposée sont évoquées.

ABSTRACT The interpretation methods used for estimating individual doses from measurement results contain some difficulties with regard to the adaptation of the metabolic models to situations encountered in practice. In essence, these difficulties concern the definition of the right parameters of the cases encountered. Moreover apart from characterised incidents, they are very often related to a lack of knowledge of the contamination routes and the chronology of the episodes. As a consequence, we were led to contemplate how the interpretation of monitoring data could be simplified by considering separately the results specific to the routes of entry and those relating to systemic contamination, i.e. after the radionuclides have been transferred to the blood. The study first develops an approach to the interpretation of the systemic contamination measurement

\footnotetext{
* Institut de protection et de sûreté nucléaire (IPSN), Département de protection de la santé de l'homme et de dosimétrie (DPHD), BP 6, 92265 Fontenay-aux-Roses Cedex.

** Institut de protection et de sureté nucléaire (IPSN), Département de protection de la santé de l'homme et de dosimétrie, Service de dosimétrie, BP 6, 92265 Fontenay-aux-Roses Cedex.
} 
results. Using the systemic contamination results and the appropriate retention and excretion functions, the values of the activity absorbed daily from the routes of entry to the blood are calculated. A day-to-day follow-up of the absorbed activity is thus made possible, providing easy-to-consult results in real time. Some applications of the method are proposed for acute tritium and uranium contamination cases, and for chronic tritium, uranium and iodine contaminations. The conditions and constraints affecting the validity of the approach proposed are discussed.

\section{Introduction}

L'exposition aux produits radioactifs en milieu professionnel reste encore bien souvent difficile à chiffrer de manière précise. L'expérience montre la convergence de divers facteurs menant à cette incertitude parfois importante : nature des produits, métabolisme individuel, chronologie et mode d'incorporation, modèle d'interprétation. Le modèle utilisé pour les calculs peut, à lui seul, générer une incertitude notable s'il n'est pas restreint à l'optimum nécessaire. L'une des façons d'aborder cette question consiste à sérier les données de la surveillance pour leur associer une modélisation répondant spécifiquement à tel ou tel maillon de la chaîne métabolique. Par exemple, analyser de manière séparée la charge systémique et celle des portes d'entrée correspond à ce type de démarche.

Les contaminations radioactives mettent généralement en jeu des quantités de produit infimes. Ainsi, les exemples donnés dans le tableau I montrent la disproportion chiffrée entre les activités considérées et les masses de matière qui leur correspondent $[5,7]$.

\section{TABLEAU I}

Correspondance entre les activités et les masses des limites annuelles d'incorporation (LAI)

Correspondence between activities and masses of the annual limits of intake (ALI)

\begin{tabular}{|c|c|c|c|c|}
\hline${ }^{3} \mathrm{H}$ & $\mathrm{D}$ & $310^{9} \mathrm{~Bq}=8,4 \mu \mathrm{g}$ & 1 & $310^{9} \mathrm{~Bq}=8,4 \mu \mathrm{g}$ \\
\hline${ }^{60} \mathrm{Co}$ & $\mathbf{Y}$ & $110^{6} \mathrm{~Bq}=2,4 \quad 10^{-2} \mu \mathrm{g}$ & 0,05 & $210^{7} \mathrm{~Bq}=4,810^{-1} \mu \mathrm{g}$ \\
\hline${ }^{90} \mathrm{Sr}$ & $\mathrm{D}$ & $710^{5} \mathrm{~Bq}=1,4 \cdot 10^{-1} \mu \mathrm{g}$ & 0,3 & $110^{6} \mathrm{~Bq}=2,010^{-1} \mu \mathrm{g}$ \\
\hline${ }^{131} \mathrm{I}$ & $\mathrm{D}$ & $210^{6} \mathrm{~Bq}=4,410^{-4} \mu \mathrm{g}$ & 1 & $110^{6} \mathrm{~Bq}=2,210^{-4} \mu \mathrm{g}$ \\
\hline${ }^{137} \mathrm{Cs}$ & $\mathrm{D}$ & $610^{6} \mathrm{~Bq}=1,9 \mu \mathrm{g}$ & 1 & $410^{6} \mathrm{~Bq}=1,2 \mu \mathrm{g}$ \\
\hline${ }^{239} \mathrm{Pu}$ & W & $210^{2} \mathrm{~Bq}=8,710^{-2} \mu \mathrm{g}$ & $10^{-3}$ & $310^{4} \mathrm{~Bq}=13,1 \mu \mathrm{g}$ \\
\hline
\end{tabular}


A l'incorporation, ces quantités abordent des surfaces épithéliales considérables : $30 \mathrm{~m}^{2}$ (expiration) et $100 \mathrm{~m}^{2}$ (inspiration) pour l'appareil respiratoire, $200 \mathrm{~m}^{2}$ pour le tube digestif [3]. Le rapport masse de produitsurface de contact et d'entrée vers la région systémique est infiniment petit. Dans ces conditions, le transfert depuis les portes d'entrée vers le sang obéit à des lois à forte connotation aléatoire, et ceci d'autant plus que le produit est peu soluble.

Il est évident que si des études métaboliques sont menées sur des animaux identiques, dans des conditions standardisées, et à des niveaux de contamination élevées, le caractère aléatoire s'estompe et on a, dans l'ensemble, l'impression d'observer des comportements similaires dont on peut tirer des lois relativement bien établies.

Il n'en va pas de même dans les conditions réelles d'exposition des personnes à cause des particularités individuelles et de la complexité des produits incriminés, souvent des mélanges aux caractéristiques physico-chimiques plus ou moins bien définies. De plus, les quantités incorporées sont faibles, inférieures aux LAI ou de quelques LAI au plus. Dans ce cas, la dispersion de la distribution initiale du produit sur les surfaces épithéliales de contact, puis le transfert vers le sang, obéissent probablement à des mécanismes dont le caractère aléatoire est très marqué. L'aspect stochastique du métabolisme de transfert prend le dessus.

Ceci peut expliquer les apparentes anomalies de comportement métabolique observées en pratique par rapport aux modèles standards. Parfois, une certaine incohérence semble se manifester et de réelles difficultés en résultent pour déterminer les niveaux de contamination.

Il paraît donc plausible d'en déduire que les transferts de la porte d'entrée vers le sang, dans les conditions habituelles de contamination, ne peuvent être définis d'une manière universelle par un modèle, d'autant plus si l'on quantifie les valeurs des paramètres de ce modèle avec un certain déterminisme. Il faut donc envisager d'aborder la modélisation en s'affranchissant le plus possible d'une règle préétablie en ce qui concerne le comportement du produit depuis son entrée jusqu'à son passage dans le sang. Il est souhaitable de traiter de manière indépendante la charge des portes d'entrée et celle de la région proprement systémique de l'organisme.

Ce commentaire sur le caractère variable du passage systémique des radionucléides est d'ailleurs valable entre individus mais aussi pour un individu donné, même si les conditions d'exposition sont apparemment semblables. Le caractère aléatoire prend, en effet, le dessus dans chaque situation particulière. Diverses observations illustrent cette constatation; à titre d'exemple, on peut citer une expérience parfaitement contrôlée quant à ses conditions de réalisation où une eau minérale naturellement riche en uranium a été administrée chez des volontaires [1]. Chez un même individu, dans des conditions expérimentales virtuellement identiques, la variabilité de l'excrétion urinaire peut atteindre un facteur 10. Une autre illustration, pour le futur immédiat, 
concerne l'exposition à des aérosols issus de combustibles à base d'oxydes mixtes $\mathrm{Pu} / \mathrm{U}$ (Mox). On sait, dès à présent, que compte tenu de la diversité des mélanges, il sera impossible de systématiser leur solubilité et leur transférabilité, et donc de définir, de manière simple, les facteurs de transfert du poumon vers le sang.

\section{Objectifs de cette étude}

L'interprétation des résultats de mesure de la contamination des personnes par des produits radioactifs est un sujet complexe et en constante évolution. Cette évolution concerne essentiellement la qualité et la limite de détection des mesures et la représentation des modèles métaboliques et dosimétriques. Par contre, la façon d'aborder l'interprétation des données de la surveillance individuelle est peu remise en question. L'idée actuelle est d'essayer de déterminer au mieux l'incorporation en utilisant des modèles métaboliques qui décrivent globalement la cinétique du radionucléide depuis son entrée dans l'organisme jusqu'à l'excrétion $[4,6]$. C'est précisément cette question qui mérite d'être reposée et il paraît intéressant d'envisager une autre approche.

Le but est d'utiliser le nombre de paramètres utiles le plus petit possible, c'est-à-dire une modélisation a minima. Ceci conduit de facto à une incertitude moindre sur les estimations des activités qui sont entrées dans l'organisme. On peut distinguer, d'un point de vue anatomo-physiologique, deux grandes régions :

- les portes d'entrée: appareil respiratoire, tube digestif, peau et muqueuses externes ;

- la partie systémique de l'organisme, concernée après entrée du produit radioactif dans le sang.

Dans la plupart des cas, la contamination systémique pose l'essentiel du problème en termes de dose et de risque pour la santé. Toutefois, pour les produits relativement insolubles, le risque aux portes d'entrée intervient de façon non négligeable.

On se propose d'aborder l'interprétation des résultats de mesure en séparant ces deux grandes régions de l'organisme, la présente étude étant consacrée à la partie systémique. Le but est d'estimer l'activité transférée des portes d'entrée vers le sang à partir des mesures de rétention et d'excrétion systémiques. Le point capital de cette approche est de traiter de manière indépendante la charge systémique et celle des portes d'entrée. Cela permet d'éviter dans les calculs l'utilisation de paramètres de transfert dont il est souvent difficile d'analyser la nature et de prouver la valeur.

Le développement futur de cette approche sera d'aborder la question du rapport entre la charge systémique et celle des portes d'entrée pour voir si ce rapport obéit vraiment à une loi assez constante ou, au contraire, si les fluctua- 
tions ne permettent pas d'établir la réalité d'une telle loi. Cet aspect est très important pour établir la crédibilité des estimations dosimétriques lorsqu'elles sont faites exclusivement sur la base de l'incorporation.

\section{Principe de l'estimation de l'activité absorbée ${ }^{1}$}

La quantité de radionucléide transférée chaque jour depuis la porte d'entrée vers le sang apporte sa contribution aux grandeurs systémiques, rétention(s) et excrétion(s), qui seront mesurées par la suite. Schématiquement, celles-ci peuvent être considérées comme la somme des activités élémentaires qui résultent des passages systémiques successifs du radionucléide.

Soient :

$A(\tau) \quad$ l'activité absorbée au jour $\tau$;

$q_{\mathrm{s}}(t-\tau+1)$ la rétention ou l'excrétion systémique qui résulte, au temps $t \geq \tau$, d'une absorption unitaire se produisant au jour $\tau$.

Les valeurs $Q(t)$ des grandeurs systémiques mesurées au jour $t$ s'écrivent : au $1^{\text {er }}$ jour : $Q(1)=q_{\mathrm{s}}(1) A(1)$

$$
\begin{aligned}
& 2^{\mathrm{e}} \text { jour: } Q(2)=q_{\mathrm{s}}(1) A(2)+q_{\mathrm{s}}(2) A(1) \\
& 3^{\mathrm{e}} \text { jour : } Q(3)=q_{\mathrm{s}}(1) A(3)+q_{\mathrm{s}}(2) A(2)+q_{\mathrm{s}}(3) A(1) \\
& 4^{\mathrm{e}} \text { jour : } Q(4)=q_{\mathrm{s}}(1) A(4)+q_{\mathrm{s}}(2) A(3)+q_{\mathrm{s}}(3) A(2)+q_{\mathrm{s}}(4) \mathrm{A}(1)
\end{aligned}
$$

soit, sous forme générale,

$$
\text { au jour } t: Q(t)=\sum_{\tau=1}^{t} q_{\mathrm{s}}(t-\tau+1) A(\tau) \quad t \geq \tau
$$

Les valeurs de $A(\tau)$ sont facilement déterminées à partir du système d'équations précédent puisqu'il y a à chaque fois une inconnue par équation. Mathématiquement, il s'agit d'une déconvolution discrète ${ }^{2}$.

La quantité de radionucléide absorbée entre deux dates $\tau_{1}$ et $\tau_{2}$ est égale à la somme des absorptions journalières qui se sont produites dans cet intervalle de temps :

$$
A\left(\text { de } \tau_{1} \grave{a} \tau_{2}\right)=\sum_{\tau_{1}}^{\tau_{2}} A(\tau)
$$

1. L'activité absorbée représente une fraction plus ou moins importante de l'activité incorporée. Cette fraction, en rapport avec la transférabilité, dépend de la nature du produit et de la voie d'entrée. Par conséquent, l'activité absorbée n'est pas directement comparable aux limites annuelles d'incorporation LAI. 2. Ces calculs sont faciles à réaliser quant au principe, mais nécessitent en pratique l'utilisation d'un outil informatique. 


\section{Avantages de cette approche}

L'interprétation des résultats de mesure telle qu'elle est préconisée à l'heure actuelle par la Commission internationale de protection radiologique (CIPR) nécessite l'élaboration de modèles métaboliques complets, de l'incorporation jusqu'aux rétentions et excrétions [5-6]. Des difficultés apparaissent en particulier lorsqu'il faut expliciter la cinétique d'entrée vers le compartiment systémique. La complexité de la modélisation s'accroît au fur et à mesure que l'on souhaite prendre en compte les connaissances les plus récentes sur les mécanismes d'épuration des organes et tissus d'entrée. Ce qui est gênant, c'est le fait que l'essentiel des valeurs attribuées aux différents paramètres n'est pas, faute d'information suffisante, personnalisé et adapté aux cas particuliers rencontrés. Les variations des paramètres sont liées à la nature des produits contaminants et aux caractéristiques propres d'un individu au plan physiologique. De plus, il n'est pas envisageable de tout modéliser, dans les moindres détails, sans paralyser le protocole d'interprétation par la prise en compte d'un trop grand nombre de facteurs. Enfin, il est clair qu'il n'est pas possible de créer un modèle pour toutes les voies d'entrée potentiellement imaginables, avec des modalités de contamination spécifiques d'un cas à l'autre. L'aspect "chronologie" de la contamination joue également un rôle très important et, hormis un incident avéré, il est rarement possible de savoir de quelle manière s'est déroulée l'incorporation.

L'approche proposée permet de s'affranchir de bon nombre de ces facteurs à problème. En fait, théoriquement, il suffit de bien maîtriser la fonction $q_{\mathrm{s}}$ qui décrit la rétention ou l'excrétion systémique après absorption d'une activité unitaire. Affiner cette fonction est un objectif que l'on peut espérer atteindre avec des moyens théoriques ou expérimentaux bien plus restreints que ceux nécessaires pour le modèle débutant dès l'incorporation. Le point important est de disposer de formes chimiques représentatives de celles sous lesquelles le radionucléide entre dans les liquides extra-cellulaires, en particulier en ce qui concerne l'état redox, la valence, ainsi que les ions ou les molécules complexantes associées.

\section{Aspects pratiques}

La réelle difficulté qui subsiste est liée au nombre de mesures $Q(t)$ dont on dispose. Pour appliquer correctement le système d'équations proposé, il faut disposer du plus grand nombre possible de mesures; or, ceci est rarement le cas, pour des raisons simplement pratiques. On peut envisager, pour certaines situations, une amélioration dès lors que tous les partenaires intervenant dans le protocole de surveillance seront convaincus du bien-fondé et de l'intérêt de la méthode. Il n'en reste pas moins vrai qu'une contrainte excessive irait à l'encontre du but recherché.

Il faut donc que le processus utilisé pour l'interprétation s'accommode d'un nombre limité de mesures et les exploite au mieux. La solution adoptée va au 
plus simple et consiste à prendre comme valeur pour $Q(t)$ celle mesurée en la gardant inchangée jusqu'à la mesure suivante, et ainsi de suite. Autrement dit, la mesure effectuée à un moment donné fait référence durant tout l'intervalle de temps qui s'écoule jusqu'à l'examen suivant.

Il résulte de ce processus que les valeurs trouvées d'absorption $A(\tau)$ sont plus des incréments mathématiques que des valeurs réelles. En effet, si les calculs effectués sur une certaine période surestiment l'absorption, il y aura, lors de l'intervalle suivant, une série de valeurs de $\mathrm{A}(\tau)$ négatives. Ce qui importe, c'est de faire la somme des $\mathrm{A}(\tau)$ sur des durées assez longues, et ce d'autant plus que les examens sont espacés. Cette sommation d'incréments, tantôt positifs, tantôt négatifs, permet à terme de contrebalancer soit une surestimation soit une sous-estimation. Finalement, l'addition algébrique des $A(\tau)$ permet d'atteindre une valeur d'absorption assez représentative de la réalité. Le processus proposé réalise en quelque sorte une autocorrection progressive, approchant la réalité tantôt par excès, tantôt par défaut. Finalement, cela revient à prendre en compte les différentes mesures un peu à la manière d'une moyenne.

Le problème qui reste posé est celui de la règle à fixer pour la fréquence des examens afin que les résultats ne soient pas entachés d'une incertitude excessive. Cela nécessite une étude théorique impliquant le couple de facteurs "périodes effectives de la fonction $q_{\mathrm{s}}$ " et "intervalles possibles de surveillance". Parallèlement, des tests pratiques sur des situations observées permettront de valider l'approche proposée.

Les exemples suivants servent à illustrer l'utilisation du protocole de calcul qui vient d'être décrit et à faire un bilan préliminaire sur les conditions et les contraintes de son application pratique.

\section{Application de la méthode à quelques cas}

\subsection{Contamination par le tritium}

Les données correspondent à une exposition accidentelle à l'eau tritiée rapportée par Snyder et al. [9]. Les résultats des mesures urinaires et la détermination de l'activité absorbée sont visualisés dans la figure 1. La fonction d'excrétion utilisée pour les calculs s'écrit :

$$
\frac{\mathrm{e}^{-\frac{0,693}{10} t}}{42000} \mathrm{~Bq} / \mathrm{ml} \text { pour } 1 \mathrm{~Bq} \text { absorbé, le temps } t \text { est en jours. }
$$

Il s'agissait d'une incorporation quasi-instantanée, ce que montre clairement la figure représentant les incréments d'activité absorbée. Au delà du 1er jour, les incréments sont virtuellement nuls. L'activité absorbée, obtenue par addition algébrique des incréments, est de l'ordre de $3,510^{9} \mathrm{~Bq}$. 
J. PIECHOWSKI, B. MENOUX
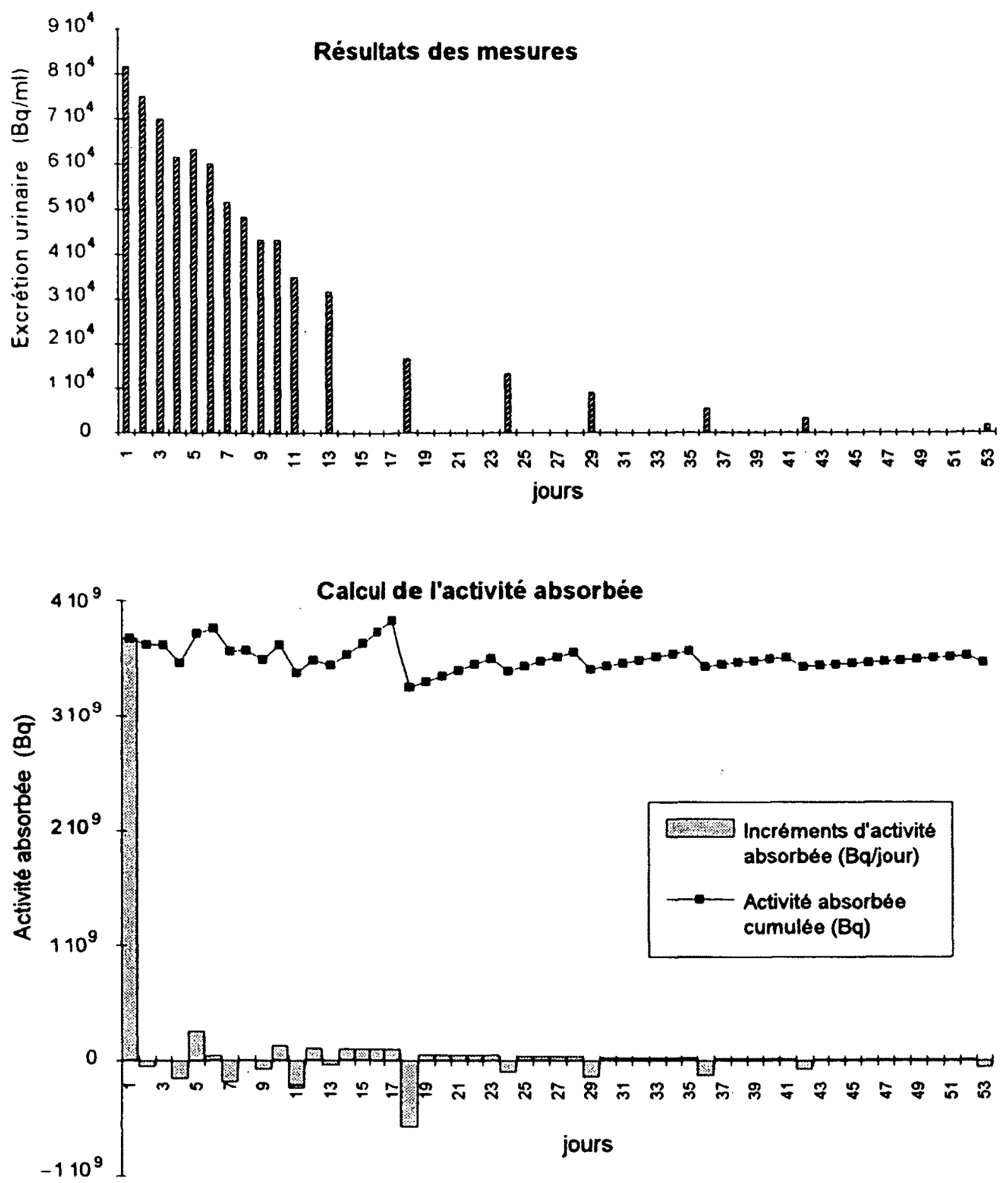

Fig. 1 - Tritium absorbé après inhalation aiguë: la quantité absorbée est estimée par déconvolution discrète à partir des mesures urinaires.

Tritium absorbed after acute inhalation : the quantity absorbed is estimated from the urine measurements by discrete deconvolution. 


\subsection{Etude expérimentale concernant l'uranium}

Une étude conduite par Harduin et al. [1] a porté sur l'absorption et l'excrétion urinaire de l'uranium présent dans une eau minérale de consommation courante. L'expérimentation fut réalisée chez quelques volontaires acceptant de boire d'une manière aiguë puis chronique des quantités bien définies de cette eau contenant $3,55 \mathrm{~Bq}$ d'uranium par litre.

Les données collectées dans le cadre de ce travail ont été reprises pour tester la méthode d'interprétation proposée. Elles sont reproduites dans les figures 2 (incorporation aiguë) et 3 (incorporation chronique), pour un même individu. Les incréments d'activité absorbée ont été calculés en utilisant la fonction d'excrétion urinaire journalière, déterminée à partir de la fonction de débit d'excrétion donnée dans la publication 54 de la CIPR [6,8]. Pour $1 \mathrm{~Bq}$ absorbé, l'excrétion en Bq/jour s'écrit :

$$
\begin{aligned}
8,04 \mathrm{e}^{-\frac{0,693}{0,25} t} & +2,9410^{-2} \mathrm{e}^{-\frac{0,693}{6} t}+7,2510^{-3} \mathrm{e}^{-\frac{0,693}{20} t} \\
& +4,8110^{-7} \mathrm{e}^{-\frac{0,693}{1500} t}+3,1910^{-6} \mathrm{e}^{-\frac{0,693}{5000} t}
\end{aligned}
$$

Il est possible de suivre la cinétique journalière d'absorption. Plusieurs remarques peuvent être faites: l'absorption se prolonge sur quelques jours après incorporation aiguë (ingestion de 21 en une journée) (Fig. 2); elle fluctue de manière non négligeable même en cas d'ingestion bien régulière d'une quantité parfaitement définie (1 1 par jour pendant 15 jours) (Fig. 3). La méthode proposée permet de suivre ces évolutions et de déterminer l'activité totale absorbée : $\sim 40 \mathrm{mBq}$ dans le cas aigu, $\sim 170 \mathrm{mBq}$ dans le cas chronique.

Toutefois, l'interprétation repose sur la qualité de la fonction d'excrétion utilisée et il convient donc d'avoir la possibilité de s'assurer de sa validité.

\subsection{Suivi d'expositions en milieu professionnel}

Des cas d'exposition chronique au tritium et à l'iode 125 servent à illustrer l'intérêt de la méthode proposée pour le suivi en routine de la contamination individuelle. Les données des mesures et les résultats de l'interprétation figurent dans les figures 4 et 5 et dans les tableaux II et III. Les fonctions utilisées

pour la déconvolution sont les suivantes [6]:
- eau tritiée (excrétion urinaire en $\mathrm{Bq} / \mathrm{ml}$ pour $1 \mathrm{~Bq}$ absorbé) : $\frac{\mathrm{e}^{-\frac{0,693}{10} t}}{42000}$ 

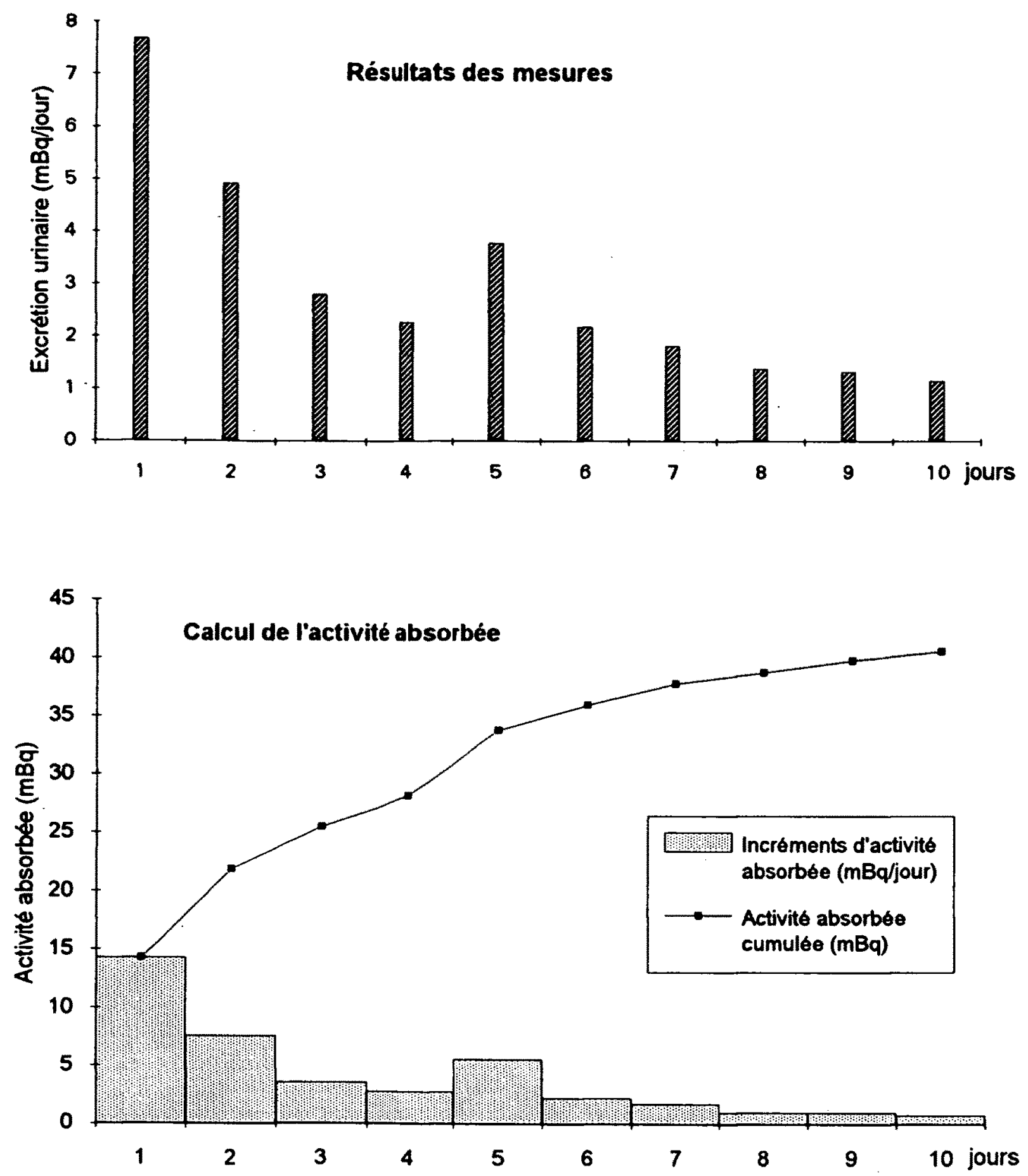

Fig. 2 - Uranium absorbé après ingestion aiguë de 7,10 Bq en 1 jour : la quantité absorbée est estimée par déconvolution discrète à partir des mesures urinaires.

Uranium absorbed after acute ingestion of $7.10 \mathrm{~Bq}$ in 1 day : the quantity absorbed is estimated from the urinary measurements by discrete deconvolution. 

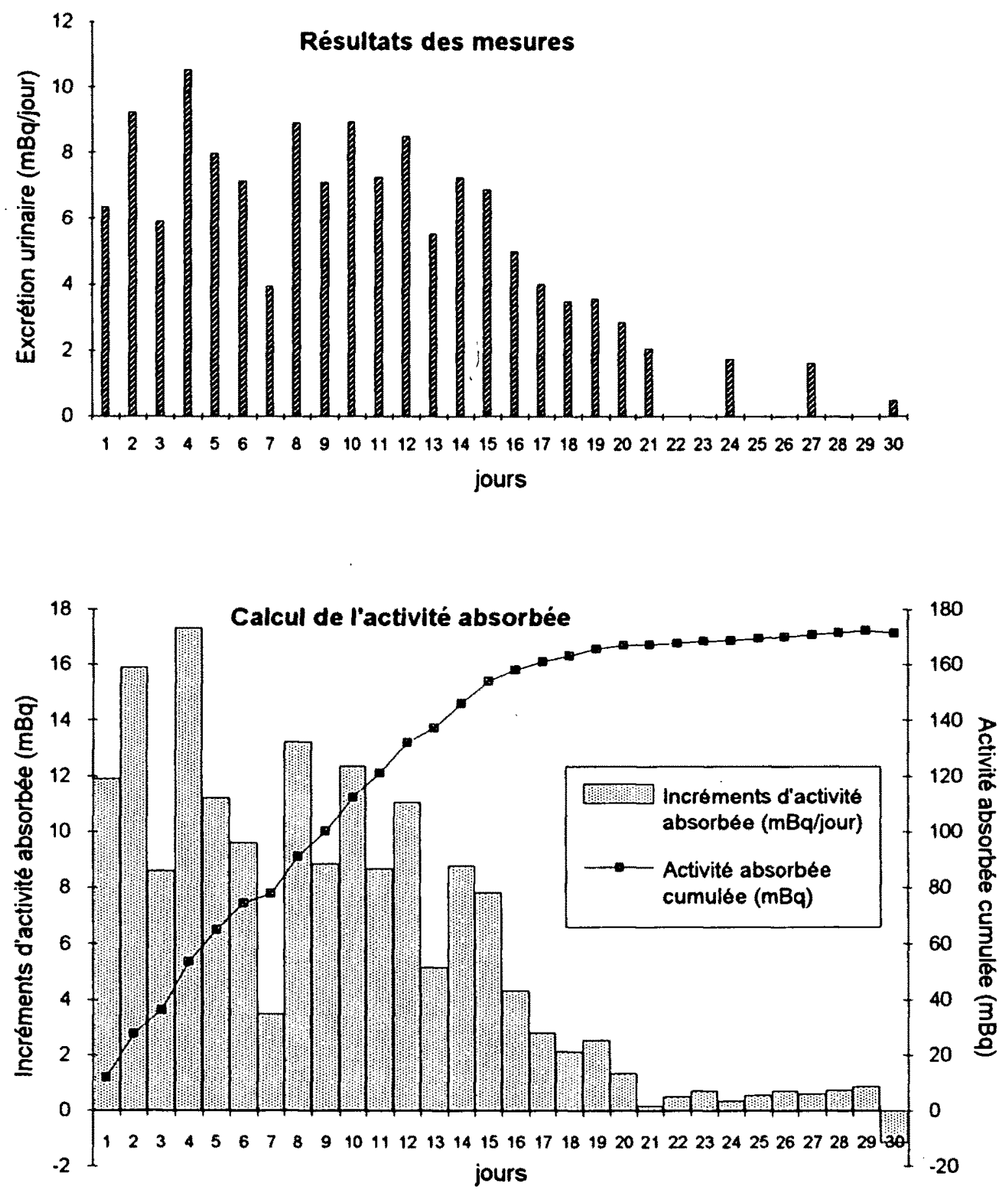

Fig. 3 - Uranium absorbé après ingestion chronique de 3,55 $\mathrm{Bq}$ par jour pendant 15 jours : la quantité absorbée est estimée par déconvolution discrète à partir des mesures urinaires.

Uranium absorbed after chronic ingestion of $3.55 \mathrm{~Bq}$ per day for 15 days : the quantity absorbed is estimated from the urinary measurements by discrete deconvolution. 
J. PIECHOWSKI, B. MENOUX

\section{Résultats des mesures}

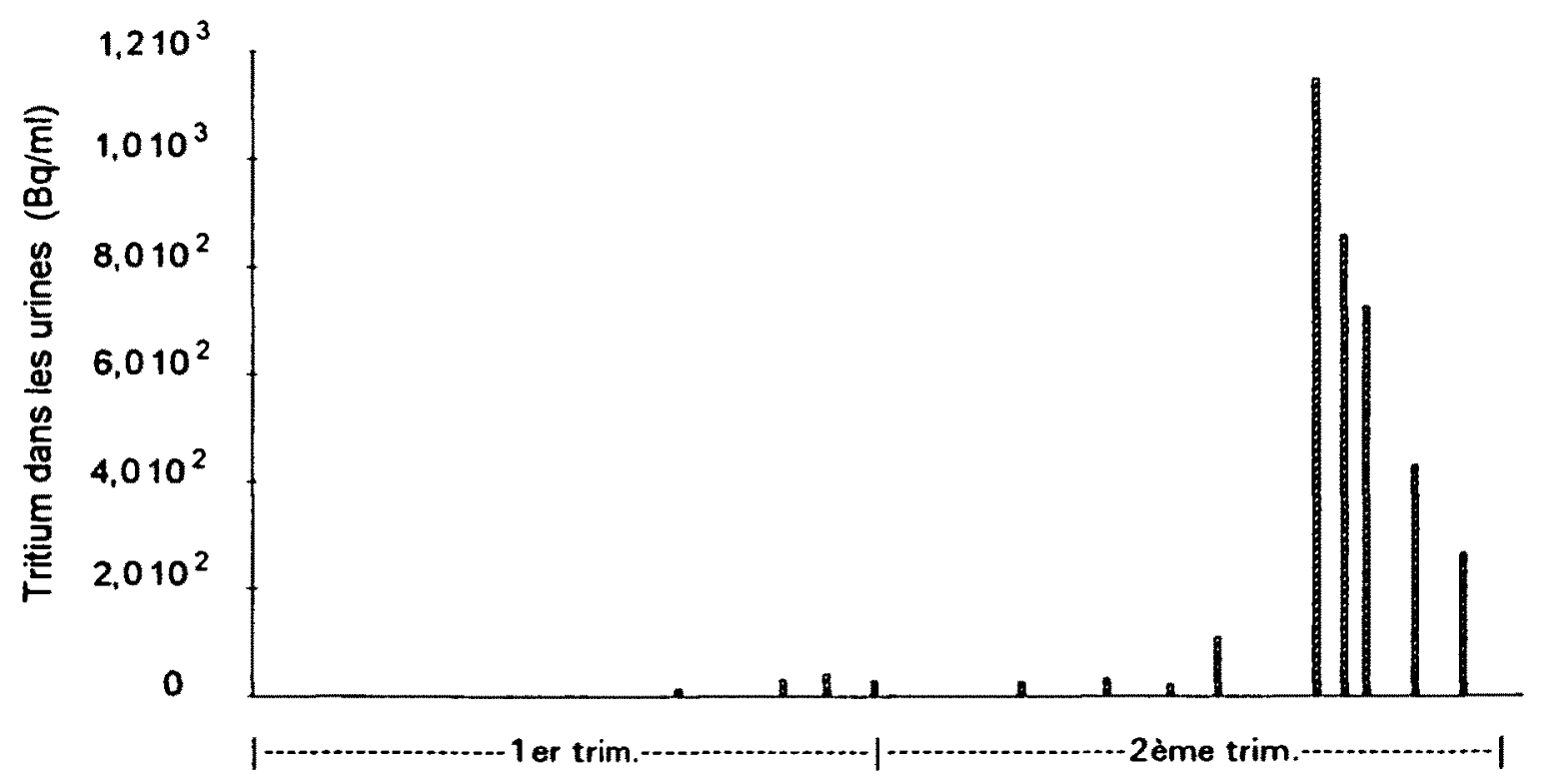

Activité absorbée cumulée (Bq)

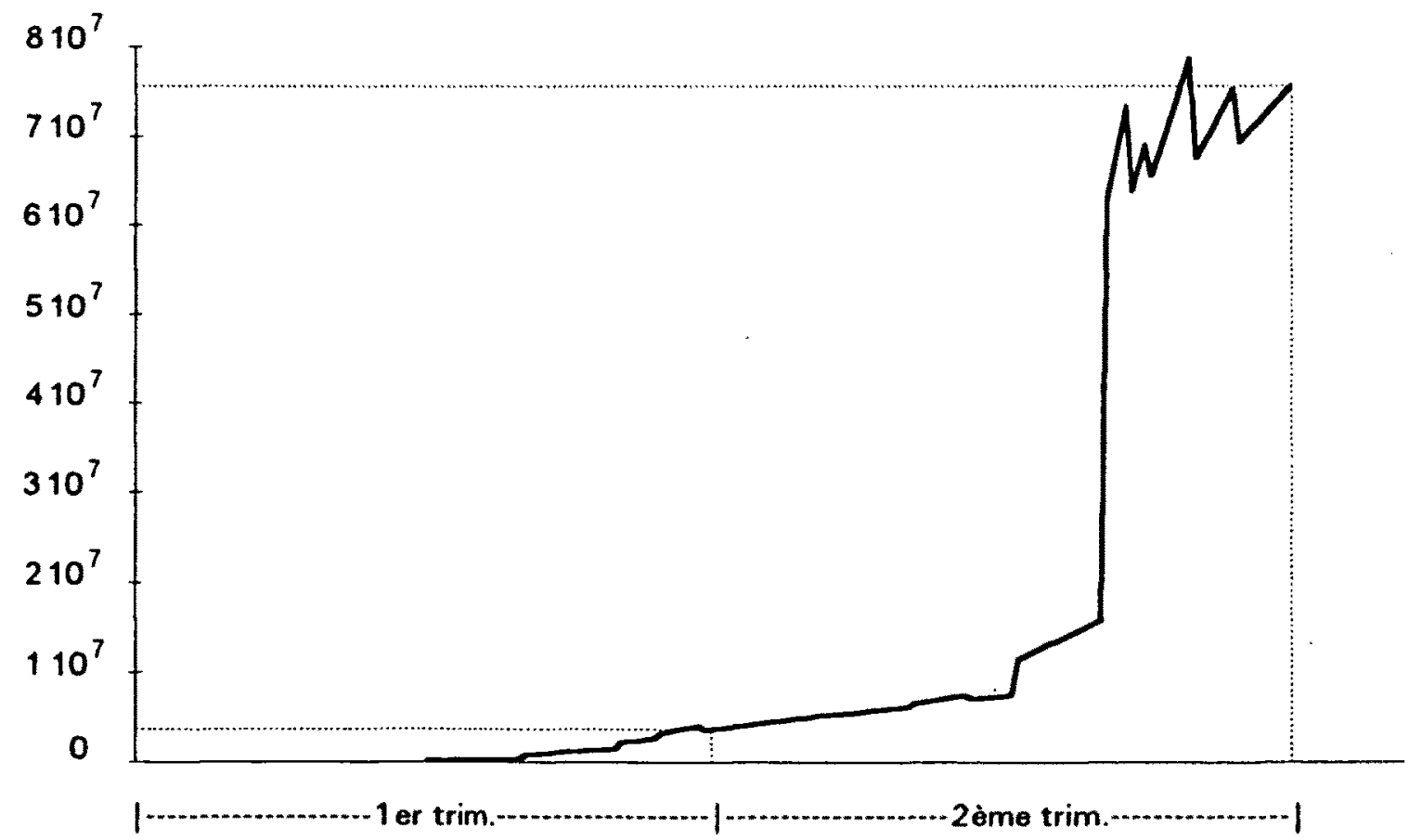

Fig. 4 - Tritium absorbé de manière chronique: la courbe représentant l'évolution de l'activité absorbée au cours du temps permet de faire un suivi régulier de l'exposition systémique (en pratique, un bilan établi sur un rythme trimestriel semble être bien adapté; voir aussi tableau II).

Chronic absorption of tritium: the curve representing the evolution of the absorbed activity over time makes it possible to carry out a regular follow-up of the systemic exposure (in practice, a quarterly summary seems to be appropriate; see also table II). 
TABLEAU II

Suivi d'une exposition au tritium - mesures de la concentration Follow-up of an exposure to tritium - urine concentration measurements

\begin{tabular}{|c|c|c|c|c|}
\hline $\begin{array}{l}\text { Dares } \\
\text { des examens }\end{array}$ & (j) & $\begin{array}{l}\text { Resulta } \\
\text { Resumes } \\
\text { esures }\end{array}$ & Activite a & $\begin{array}{l}\text { ég }(\mathrm{Bq}) \\
\text { cumul annuel }\end{array}$ \\
\hline $14 / 02 / 1989$ & 1 & 3 & \multirow{6}{*}{$1^{\text {er }}$ trim. $3,6510^{6}$} & \multirow{15}{*}{$\begin{array}{c}\text { Année } 1989 \\
7,5710^{7}\end{array}$} \\
\hline $16 / 02 / 1989$ & 3 & 3 & & \\
\hline 02/03/1989 & 17 & 15 & & \\
\hline $17 / 03 / 1989$ & 32 & 30 & & \\
\hline 23/03/1989 & 38 & 42 & & \\
\hline $30 / 03 / 1989$ & 45 & 29 & & \\
\hline 20/04/1989 & 66 & 27 & \multirow{9}{*}{$2^{e}$ trim. $7,2010^{7}$} & \\
\hline 02/04/1989 & 78 & 34 & & \\
\hline $11 / 05 / 1989$ & 87 & 23 & & \\
\hline $18 / 05 / 1989$ & 94 & 110 & & \\
\hline $01 / 06 / 1989$ & 108 & 1150 & & \\
\hline 05/06/1989 & 112 & 860 & & \\
\hline 08/06/1989 & 115 & 725 & & \\
\hline $15 / 06 / 1989$ & 122 & 430 & & \\
\hline $22 / 06 / 1989$ & 129 & 266 & & \\
\hline
\end{tabular}

Les quantités absorbées sont estimées par déconvolution discrète à partir des mesures urinaires; on remarquera l'incident de contamination survenu fin mai. Un cumul trimestriel de l'activité absorbée permet de suivre l'exposition au cours du temps.

- iode 125 (rétention dans la thyroïde en $\mathrm{Bq}$ pour $1 \mathrm{~Bq}$ absorbé) :

$$
\left(-3,28210^{-1} \mathrm{e}^{-\frac{0,693}{0,243} t}+1,79010^{-2} \mathrm{e}^{-\frac{0,693}{11,32} t}+3,10310^{-1} \mathrm{e}^{-\frac{0,693}{118,9} t}\right) \mathrm{e}^{-\frac{0,693}{60,14} t}
$$

L'absorption journalière a été cumulée par trimestre et par an. Il est ainsi possible de suivre de manière continue l'activité absorbée et de la répertorier sous forme d'un bilan régulier, facile à consulter. Il faut, toutefois, veiller à ce que le rythme des examens radiotoxicologiques soit suffisant pour garantir la validité des résultats. 


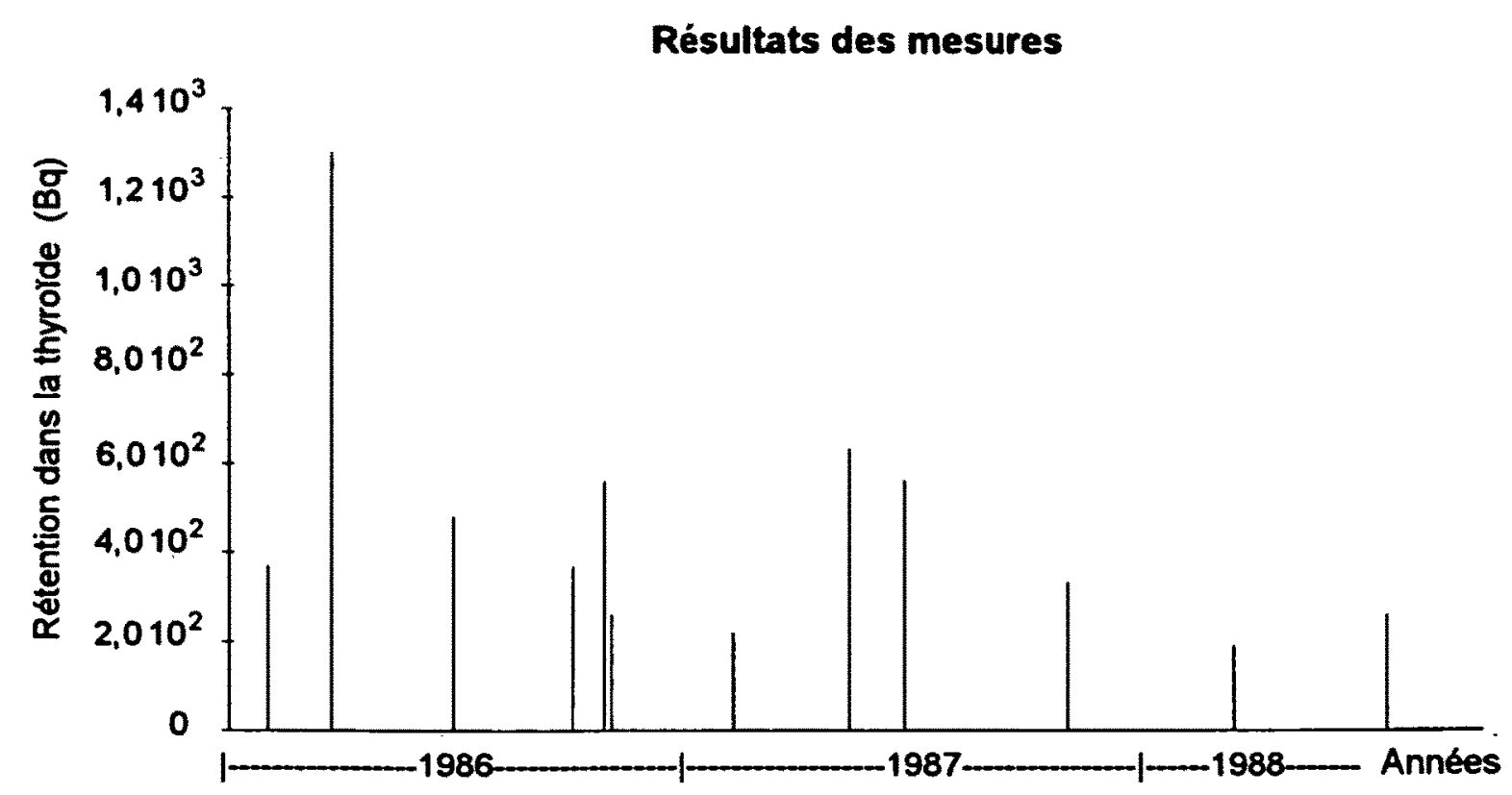

\section{Activité absorbée cumulée (Bq)}

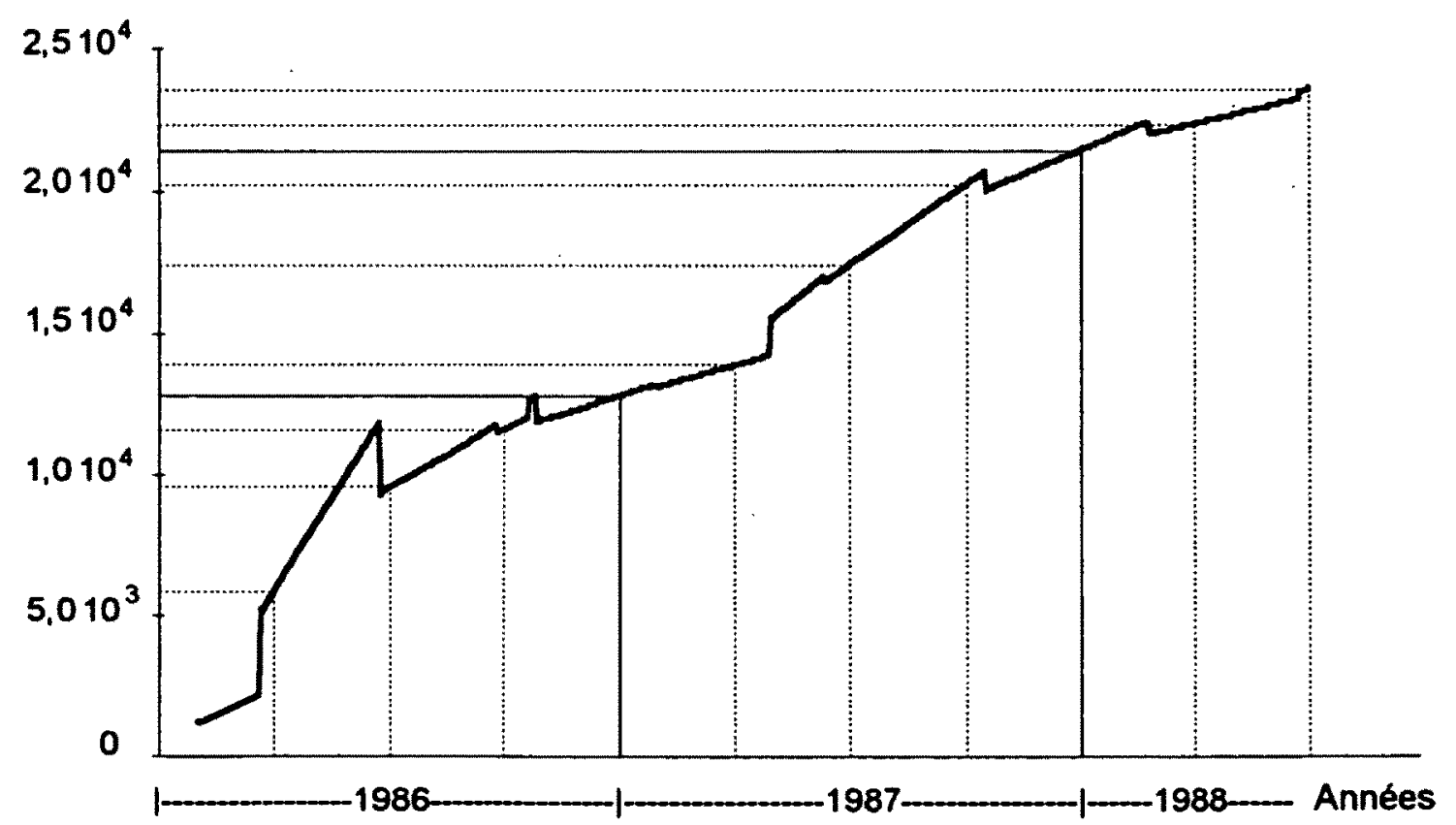

Fig. 5 - 125 I absorbé de manière chronique : la courbe représentant l'évolution de l'activité absorbée au cours du temps permet de faire un suivi régulier de l'exposition systémique (en pratique, un bilan établi sur un rythme trimestriel semble être bien adapté; voir aussi tableau III).

Chronic absorption of $125 I$ : the curve representing the evolution of the absorbed activity over time makes it possible to carry out a regular follow-up of the systemic exposure (in practice, a quaterly summary seems to be appropriate; see also table III). 
TABLEAU III

Suivi d'une exposition à l'iode 125 - mesures par comptage de la thyroïde Follow-up of an exposure to iodine 125 - measurements by thyroid counting

\begin{tabular}{|c|c|c|c|c|}
\hline \multicolumn{5}{|c|}{ 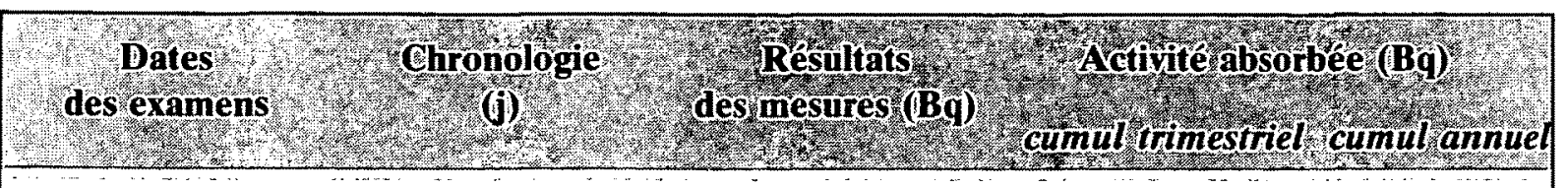 } \\
\hline $31 / 01 / 1986$ & 1 & 370 & & \multirow{6}{*}{$\begin{array}{c}\text { Année } 1986 \\
1,28 \quad 10^{4}\end{array}$} \\
\hline $21 / 03 / 1986$ & 50 & 1296 & $1^{\text {er }}$ trim. $\left.5,8510^{3}\right)$ & \\
\hline 23/06/1986 & 144 & 481 & $2^{\mathrm{e}}$ trim. $3,7310^{3}$ & \\
\hline $24 / 09 / 1986$ & 237 & 370 & 3e trim. $2,0210^{3}$ & \\
\hline $22 / 10 / 1986$ & 265 & 555 & & \\
\hline 28/10/1986 & 271 & 259 & $4^{e}$ trim. $1,2110^{3}$ & \\
\hline 29/01/1987 & 364 & 222 & $1^{\text {er }}$ trim. $\left.1,0910^{3}\right)$ & \multirow{4}{*}{$\begin{array}{c}\text { Année } 1987 \\
8,6210^{3}\end{array}$} \\
\hline $29 / 04 / 1987$ & 454 & 629 & $2^{\mathrm{e}}$ trim. $3,5010^{3}$ & \\
\hline $11 / 06 / 1987$ & 497 & 555 & $3^{\mathrm{e}}$ trim. $2,8010^{3}$ & \\
\hline $16 / 10 / 1987$ & 624 & 333 & $4^{\mathrm{e}}$ trim. $\left.1,2310^{3}\right)$ & \\
\hline $24 / 02 / 1988$ & 755 & 185 & $1^{\mathrm{er}}$ trim. $0,8710^{3}$ & \multirow{2}{*}{$\begin{array}{c}\text { Année } 1988 \\
2,1210^{3}\end{array}$} \\
\hline $23 / 06 / 1988$ & 875 & 259 & $2^{\mathrm{e}}$ trim. $1.2510^{3}$ & \\
\hline
\end{tabular}

Les quantités absorbées sont estimées par déconvolution discrète à partir des mesures urinaires ; l'exposition paraît être chronique. Un cumul trimestriel de l'activité absorbée permet de suivre l'exposition au cours du temps.

\section{Conclusion}

L'estimation de la contamination systémique directement à partir des résultats de mesure constitue une nouvelle approche dans le domaine de la dosimétrie interne. Elle présente l'avantage d'éviter les aléas liés à l'interprétation des résultats basés sur le calcul de l'incorporation. Ces aléas sont importants. Ils portent sur les voies et les épisodes de contamination, ainsi que sur la cinétique suivie par les radionucléides depuis les portes d'entrée jusqu'au sang. L'expérience a largement prouvé qu'en ce domaine la situation ne peut guère s'améliorer. La méthodologie proposée limite les fluctuations d'interprétation susceptibles d'être observées lorsque les données du suivi radiotoxicologique sont soumises à divers experts $[2,10]$. En effet, une seule fonction intervient pour le calcul, en l'occurrence la fonction reliant la charge systémique aux résultats de mesure.

En pratique, la nouvelle approche apparaît ainsi plus fiable pour déterminer l'activité entrée dans le compartiment sanguin. Elle pourrait être appliquée en complément de la pratique actuelle, et même s'y substituer à l'avenir lorsqu'auront été résolues les questions portant sur le rythme de la surveillance et la détermination de l'activité présente au niveau des portes d'entrée. 
La dosimétrie se rapportant à l'activité absorbée est dès à présent possible en utilisant les facteurs de dose appropriés $(\mathrm{Sv} / \mathrm{Bq}$ absorbé). Par contre, celle des portes d'entrée suppose un développement ultérieur de la méthode.

\section{RÉFÉRENCES}

[1] HARDUIN J.C., ROYER Ph., PIECHOWSKI J. - Uptake and urinary excretion of uranium after oral administration in man. In : Intakes of radionuclides, Bath, 13-17 September 1993. Radiat. Prot. Dosimetry, 1994, 53(1/4), 245-248.

[2] HUI T.E., LOESCH R.M., RADDATZ C., FISHER D.R., McDONALD J.C. - An internal dosimetry intercomparison study. Health Phys., 1994, 67 (3) 217-225.

[3] INTERNATIONAL COMMISSION ON RADIOLOGICAL PROTECTION (ICRP) Report of the Task group on reference man (ICRP Publication 23). Oxford : Pergamon Press, 1975.

[4] INTERNATIONAL COMMISSION ON RADIOLOGICAL PROTECTION (ICRP) General principles of monitoring for radiation protection of workers (ICRP Publication 35). Oxford : Pergamon Press, 1982.

[5] INTERNATIONAL COMMISSION ON RADIOLOGICAL PROTECTION (ICRP) Limits for intakes of radionuclides by workers (ICRP Publication 30). Oxford : Pergamon Press. Part 1 and supplement to Part 1, 1979; Part 2 and supplement to Part 2, 1980-1981; Part 3 and supplement to Part 3, 1981-1982 ; Part 4, 1988.

[6] INTERNATIONAL COMMISSION ON RADIOLOGICAL PROTECTION (ICRP) Individual monitoring for intakes of radionuclides by workers : design and interpretation (ICRP Publication 54). Oxford: Pergamon Press, 1988.

[7] PIECHOWSKI J., LE TOHIC J. - Correspondance entre l'activité et la masse pour les limites annuelles d'incorporation par inhalation en milieu professionnel. Rapport CEAIPSN/DPS 84/04, 1984.

[8] PIECHOWSKI J., MENOUX B. - Rétention et excrétion des radionucléides après incorporation par inhalation chez l'homme adulte. Rapport CEA-R-5266, 1984.

[9] SNYDER W.S., FISH B.R., BERNARD S.R., FORD M.R., MUIR J.R. - Urinary excretion of tritium following exposure of man to HTO - a two exponential model. Phys. Med. Biol., $1988,13(4), 547-559$.

[10] WINDHAM J.R., KEARFOTT K.J., MIS F.J. - Discrepancies in committed effective dose equivalents calculated using U.S. Nuclear regulatory commission regulatory guide 8.34. Health Phys., 1994, 67(5), 486-494. 